\title{
THE EFFECTS OF ALTERATIONS TO UPPER LIMB INERTIAL PROPERTIES ON VERTICAL POINT-TO-POINT MOVEMENT
}

\author{
A Thesis \\ Presented to \\ the Faculty of California Polytechnic State University, \\ San Luis Obispo
}

\author{
In Partial Fulfillment \\ of the Requirements for the Degree \\ Master of Science in Kinesiology
}

by

Adrian Kiyohisa Hongo

March 2009 
(C) 2009

Adrian Kiyohisa Hongo

ALL RIGHTS RESERVED 
TITLE:

AUTHOR:

DATE SUBMITTED:

COMMITTEE CHAIR:

COMMITTEE MEMBER:

COMMITTEE MEMBER:
THE EFFECTS OF ALTERATIONS TO UPPER LIMB INERTIAL PROPERTIES ON VERTICAL POINT-TO-POINT MOVEMENT

Adrian Kiyohisa Hongo

March 2009

Robert D. Clark, PhD

Kellie Green Hall, PhD

Ann Yelmokas McDermott, PhD 


\begin{abstract}
The Effects of Alterations to Upper Limb Inertial Properties on Vertical Point-to-Point Movement

Adrian Kiyohisa Hongo
\end{abstract}

Purpose: Several studies have examined intralimb coordination between the shoulder and elbow joints during target-oriented movements. These studies have observed consistent patterns in coordination despite changes in movement variables such as speed, direction, and inertia. Researchers used intersegmental dynamics to quantitatively analyze these patterns between shoulder and elbow joints while systematically changing values of these movement variables. Some studies have examined central nervous system adaptations to inertial changes at the elbow and entire arm during a movement, but none have examined inertial changes to the upper limb. Methods: Five male and five female participants aged 27 to 39 years (mean age $=33 \pm 4.3$ standard deviation) performed a maximal speed, point-to-point, reversal hand movement in the sagittal plane with and without a $2.2 \mathrm{~kg}$. weight attached to their dominant, right upper arm. To determine the effects of the added mass, dependent t-tests were performed on elbow and shoulder peak muscular torques generated during the reversal region of the movement. Results: A significant increase in shoulder joint torque $(p<0.05)$, a significant increase in movement time $(p<0.05)$ and a non-significant decrease in elbow muscular torque $(p=0.1074)$ was shown to achieve the movement objective with the added weight. Conclusions: While future studies may result in more conclusive findings, this study showed a pattern of increased shoulder torque and decreased elbow torque due to the added inertia. Larger shoulder torque was needed to overcome the added inertia and 
move at high speed. As a result of the higher shoulder force, interaction torque at the elbow increased, and a reduction in elbow torque was needed to control the hand path and accurately hit the targets. This pattern supports Bernstein's proposal that passively arising phenomena (i.e., interaction torque) is exploited during multi-segment movement. 


\section{ACKNOWLEDGEMENTS}

I’d like to sincerely thank Dr. Robert D. Clark (Kinesiology Department, Cal Poly, San Luis Obispo) for committing his creative support and sustained energy over the

duration of this project. Dr. Derek Marks (Kinesiology, St. Mary’s College, Moraga) was also a constant inspiration for his guidance and research expertise. I thank the participants in this study for their unwavering effort to give 110\%. Most importantly, I want to give thanks to all friends and family who pushed me in more ways than one to bring this project to its completion. 


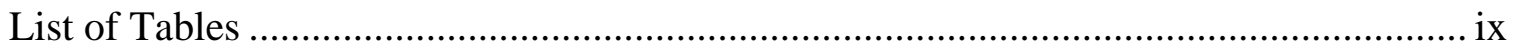

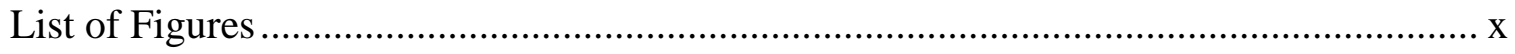

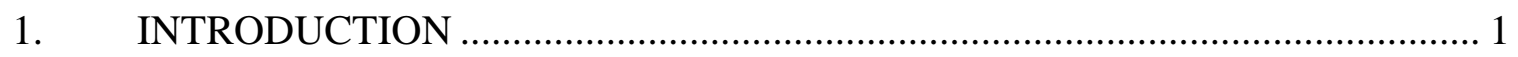

Background of the Study ....................................................................... 1

Statement of the Purpose ……………….................................................. 6

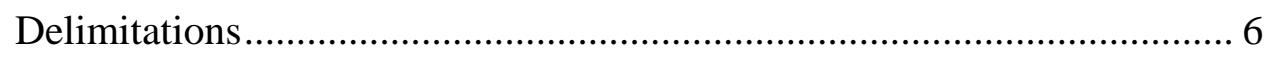

Assumptions......................................................................................... 7

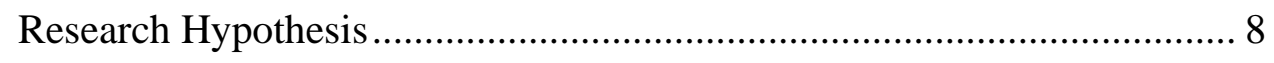

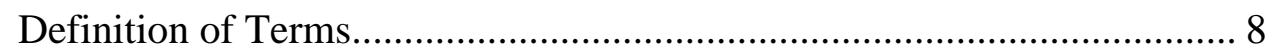

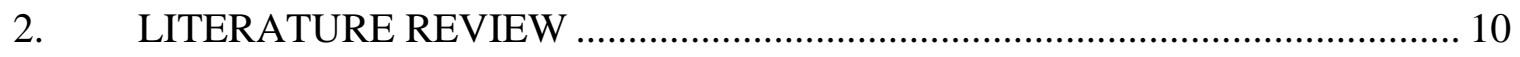

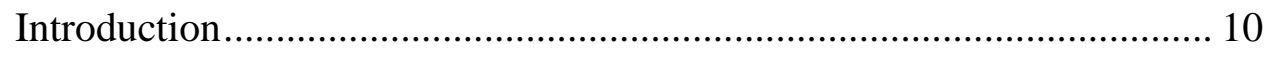

Theories explaining intralimb coordination............................................... 10

Analysis of intralimb coordination using intersegmental dynamics......... 13

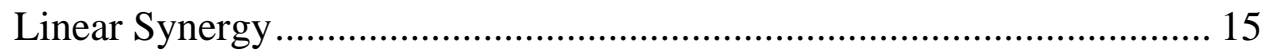

Intralimb coordination in individuals with pathologies ............................. 18

Hand dominance .................................................................................... 21

Studies that alter inertial properties during the reaching movement ........ 23

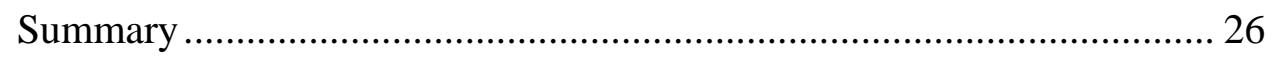

3. METHODS AND PROCEDURES ……………….................................... 28

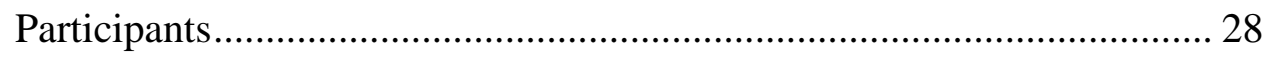

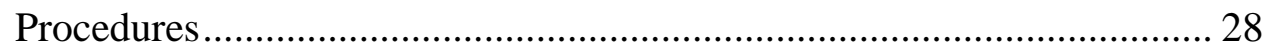


Data Computations...................................................................... 30

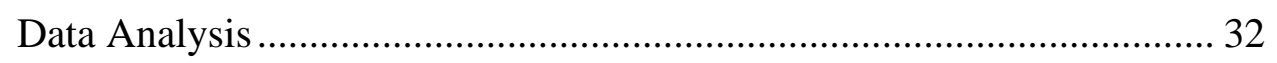

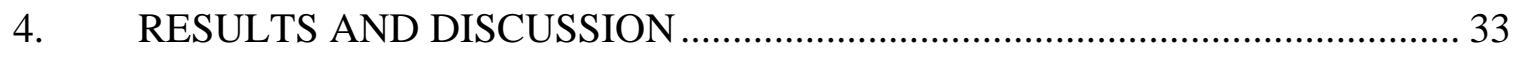

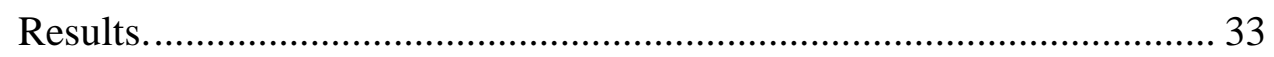

5. SUMMARY, CONCLUSIONS, AND RECOMMENDATIONS...................... 36

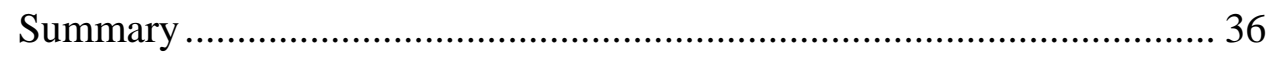

Conclusions .............................................................................. 36

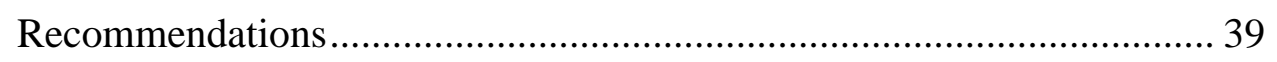

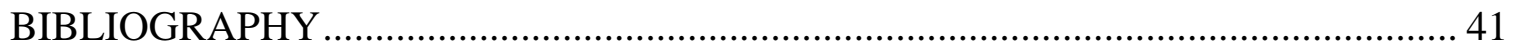

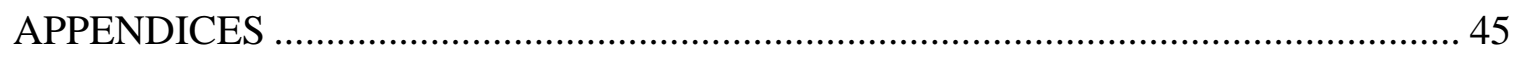

A. Informed Consent Form ............................................................... 45

B. Pre-Experiment, Sports Participation Survey ..................................... 48

C. Inverse Dynamics Equations used to Convert

Kinematic Data to Joint Torques .................................................... 49

D. Mean Values of Each Participant's Data ............................................ 51

E. Raw Data from Each Participant.................................................... 52

F. Statistical Results of Dependent t-tests............................................ 56 


\section{LIST OF TABLES}

Table

Page

1. Mean Torque and Time Data (Mean \pm Standard Deviation)

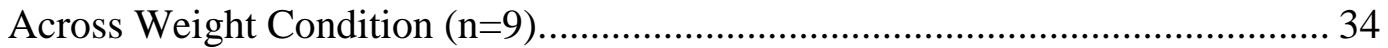




\section{LIST OF FIGURES}

Figure Page

1. Experimental Setup of Participant, Barrier,

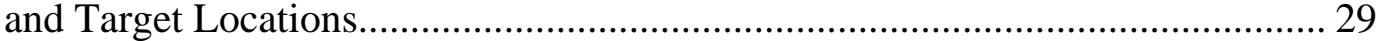

2. Orientation of the Shoulder and Elbow Angles

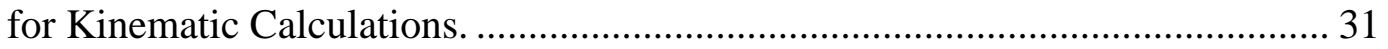

3. Typical Joint Angle and Torque Profiles Normalized

Across Each Weight Condition For One Participant. .......................................... 34 


\section{CHAPTER 1}

\section{INTRODUCTION}

\section{Background of the Study}

Several theories exist concerning the organization of motor control during coordinated, multi-segment movement in humans. These theories attempt to reveal the mechanisms that govern the complex interaction between adjacent limb segments to produce an efficient, accurate, and reproducible movement across different directions, speeds, and other movement parameters. Beginning with Bernstein’s work (1967) which gave insight into the general basis of multi-segment, coordinated movement, other developments in motor control, biomechanics, and neurology in the past few decades have contributed to understanding the control of motor patterns such as walking, handwriting, throwing, and reaching (e.g., Schmidt \& Lee, 2005). During these motor tasks, movement of one limb has been shown to affect movement of adjacent limbs, and control of the muscles across each joint exhibit a high degree of complexity that, at the time of Bernstein's work (1967), had not been fully explained. The question of interest in this area of research is whether the central nervous system (CNS) uses specific strategies or rules to create energy-efficient, reproducible movement despite the complex interaction between limb segments.

Single-joint coordination has been extensively studied and explained by theories like the equilibrium point model (Crossman \& Goodeve, 1963/1983) and the impulsetiming hypothesis (Schmidt \& Lee, 2005). However, many everyday movements are performed by the coordination of several body segments and joints. Examples include the phalanges and metacarpals during handwriting; the spine, neck, and hips when 
leaning forward; the ankle, hip, and knees during walking. As the number of joints used in a movement increase, so does the complexity and difficulty of the control required by the CNS.

The coordination of multi-limb movement varies based on environmental constraints and the goals of the movement task (Schmidt \& Lee, 2005). Control of lower body movement involves gross, less accurate positioning than the upper body and requires movement planning of both legs for stabilizing body weight. Arm movements require no weight bearing positioning, demands a higher degree of spacial and temporal precision, and, in the cases of grasping and object manipulation, presents additional complexity in the programming of coordinated movement.

The reaching movement has been studied extensively and can be categorized as discrete (Schmidt \& Lee, 2005), goal-oriented, and a closed skill (Poulton, 1957). It is different than swinging the legs during walking, which is a reflexive, continuous motor pattern characterized by a loading stance phase and a non-loaded swing phase. Throwing an object is a discrete task and its goal is a non-zero velocity of the hand. Reaching is characterized by zero-velocity start and finish positions, its goal to move the end effector with a high degree of endpoint accuracy. While the movements just mentioned are functionally different, all require coordination of several joints and the muscles that span across them to produce the desired goal.

From a biomechanical approach, the arm can use all the degrees of freedom during the reaching movement: three in the shoulder, two in the elbow, and three at the wrist. This translates into an array of possible joint movement combinations that can result at the final reach destination. Furthermore, when extending your finger to press an 
elevator button, several more degrees of freedom are introduced at each phalangeal joint to increase the level of motor pattern complexity and coordination needed to perform the simple pointing movement smoothly and accurately. At the physiological level, each joint along the upper arm has several different muscles facilitating its control, with each muscle simultaneously undergoing different phases of its length-tension, viscoelastic, and force-velocity properties. Some muscles like the biceps brachii and triceps have more than one head with different lengths, line of action, and may span both the shoulder and elbow joints. Tendons, ligaments, and other connective tissues present more viscoelastic considerations. Also, the reflexive physiology in muscle spindles and Golgi tendon organs at each joint provide more systems to control during movement. These biomechanical and physiological factors outline the complexity involved in controlling such a seemingly simple movement as in reaching from point to point.

Given the high number of degrees of freedom involved during the CNS’ control of a movement, researchers such as Bernstein (1967), Almeida, Corcos, and Hasan (2000), and Gottlieb, Song, Hong, and Corcos (1996a) hypothesized that organizing principles exist to reduce the complexity of a simple movement like reaching. Furthermore, when generating a goal-oriented movement like reaching, parameters such as speed and direction continually change which results in concomitant alterations to limb inertial properties. Regardless of these increased demands, most individuals typically reach in a straight or gradual curved path, and with a smooth velocity profile. Not surprisingly, these kinematic properties were found to be invariant in reaching movements (Abend et al., 1982). 
While extrinsic motor control strategies (i.e., exhibit kinematic patterns) have shown some merit in the reproducibility of hand trajectory during a reaching movement (Abend et al., 1982), another branch of research has sought to understand how the CNS plans and controls such a trajectory by examining the underlying kinetic variables. Intrinsic motor control strategy is characterized by the analysis of joint torque amplitude and timing between joints. This analysis evaluates the interactions between limb segments and is computed by mathematical models representing rigid body mechanics, otherwise known as intersegmental dynamics (Gottlieb, Song, Almeida, Hong \& Corcos, 1997).

The study of intersegmental dynamics during multi-joint movement has revealed patterns in torque production both between and within individual joints (Schneider, Zernicke, Schmidt, \& Hart, 1989). The process of computing joint interactions begins with the collection of kinematic data (e.g., joint position, velocities, and accelerations), which are readily available via motion analysis equipment. The resultant joint kinematics are used with limb mass and inertial assumptions in a mathematical process termed “inverse dynamics” to compute the torque at the joint in question. In this type of analysis, the limbs in motion are modeled as rigid links with frictionless joints, and representative equations of motion (i.e., dynamical equations) relate joint torques to forces of gravity, movement accelerations and velocities, and forces created by adjacent segments. Through investigation of these torques and their interrelationship in studies that vary movement parameters (e.g., speed and direction), researchers have found that an internal representation of the motor pattern (i.e., the patterned manipulation of joint torques) is a precise form of CNS motor control. 
In an early study that involved a comprehensive analysis of joint torques, Schneider et al. (1989) evaluated the intersegmental dynamics of a maximal speed, sagittal-plane reaching movement. Schneider et al. supported Bernstein’s work (1967) regarding the development of coordinated movement thru the identification and examination of different types of torques as modeled in the dynamics equations. These torques were analyzed during various phases of the reaching movement and include: 1) net joint torque, the sum of all torques acting at the joint; 2) gravitational torque, a passively acting torque (i.e., not created by the CNS, but rather an externally applied force) resulting from gravity's action on each segment's center of mass; 3) interactive or interaction torque, a passive torque that develops as a mechanical interaction between adjacent limb segments undergoing velocities and accelerations; and 4) muscle torque, a residual equation term representing the torque generated from both active muscle contraction and passive action of ligaments, tendons, and other viscoelastic tissues. As noted by Gottlieb et al. (1997), this muscle torque is the combination of all these factors, and no single muscle contribution can be uniquely identified. Mathematically, this torque is found by subtracting the other torques from net muscle torque.

With regards to the works previously discussed, the reaching movement has been described either extrinsically with kinematics, or intrinsically with joint torques. To determine if certain invariant features of intralimb coordination exist, researchers have examined hand trajectories during reaching and grasping movements, analyzed multisegment movement across different parameters such as movement speed, direction, and loading conditions, observed changes in muscle activation patterns following practice, 
and compared the endpoint errors of point-to-point movements following positional and inertial perturbations exerted on the limb.

\section{Statement of the Purpose}

A large amount of research has been performed to explain the intrinsic features of intralimb coordination between the elbow and shoulder joints during a point-to-point reaching movement. However, only a few studies have investigated the effects of altering limb inertia on the coordination between the two joints (Sainburg et al., 1999; Sainburg, 2002; Debicki \& Gribble, 2004). Of these studies, none have investigated the effects of altering upper limb inertia. Because the upper arm is the primary mover of the system, it is of interest to determine if and how the CNS adapts to the increased inertia, and if previously discovered motor control patterns are maintained. Thus, the purpose of this study is to investigate the effects of altering upper arm inertia on intralimb coordination and contribute to the existing body of research. A secondary purpose of this study is to gain experience in motion analysis using intersegmental dynamics. The information gathered in this study may contribute to a better framework for modeling motor control theories that describe discrete, upper-body, multi-segment movement. It may be applied to strategies for physical rehabilitation programs, overcoming motor learning deficiencies, and help to treat the cases of some disabilities.

$\underline{\text { Delimitations }}$

This study was delimited by the following parameters.

1. Ten right-hand dominant individuals who reside in San Luis Obispo County were tested following their informed consent. 
2. Participants performed maximal speed, sagittal plane, reversal reaching movements with and without a $2.2 \mathrm{~kg}$ mass attached above the elbow joint.

3. The testing and data collection were performed from August to September 2008 in the biomechanics laboratory located on the first floor of the Kinesiology building at California Polytechnic State University, San Luis Obispo.

\section{$\underline{\text { Assumptions }}$}

This study was based on the following assumptions.

1. All the participants tested were accurate in their acknowledgement of dominant right hand control.

2. All the participants did not have any musculoskeletal disorders, injuries, or other conditions that would affect their movement.

3. Each trial used in the data analysis was performed at the participants' best effort to achieve maximum speed.

4. Laboratory conditions such as lighting, temperature, humidity, and noise were assumed not to affect the participants’ performance during testing. 


\section{$\underline{\text { Research Hypothesis }}$}

Movement times were expected to be slower during trials with the mass than without the added mass. With the added mass, torque patterns during the fastest trials will differ than those without the inertial addition. Like other research, coordination will be exhibited between the elbow and shoulder to compensate for the added inertia and achieve the movement goal. To evaluate these hypotheses, the following null hypotheses were developed:

- Ho = The addition of weight to the upper arm will have no effect on the linear synergy between shoulder and elbow torques.

- $\quad H o=$ There will be no change in movement duration between weight and no weight conditions.

- $\quad$ Ho $=$ There will be no difference in peak muscular shoulder torque between weight and no weight conditions.

- $\quad$ Ho $=$ There will be no difference in peak muscular elbow torque between weight and no weight conditions.

\section{$\underline{\text { Definition of Terms }}$}

The following terms are defined as used in this study.

Degree of freedom (DOF). Describes an independent displacement or rotation that defines the position and orientation of a body in space (Kreighbaum \& Barthels, 1996).

Equilibrium point theory. Movement control theory that suggests muscles spanning a joint act as mass springs. For a given amount of stiffness in the agonist and antagonist muscles at any position, the joint would be stabilized, specifying an 
equilibrium point. Movement occurs when the equilibrium point changes as desired during the planning of the motor pattern (Schmidt \& Lee, 2005).

Feedforward control. Motor control strategy that incorporates proprioceptive and/or anticipatory feedback into the adaptation to a preprogrammed movement (Schmidt \& Lee, 2005).

Intersegmental dynamics. The study of mechanical interactions acting between limb segments during movement. Computed using equations of motion and results in forces and moments generated to produce the movement (Gottlieb et al., 1997).

Dynamics. The study of factors associated with systems in motion (Kreighbaum \& Barthels, 1996).

Kinematics. The study of temporal and spatial factors in the motion of a system (Kreighbaum \& Barthels, 1996).

Kinetics. The study of the forces acting on a system (Kreighbaum \& Barthels, 1996).

Linear synergy. A synchronous, linear relationship between shoulder and elbow torques proportionally related by a constant value (Gottlieb et al., 1997). 


\section{CHAPTER 2}

\section{LITERATURE REVIEW}

$\underline{\text { Introduction }}$

The following discussion will review studies that examine intralimb coordination and the intersegmental dynamics underlying the control of planar point-to-point hand movement. First, an overview of biomechanics and motor control research involving intralimb coordination that show how humans intrinsically plan and implement the reaching movement is presented. Second, studies that analyze intersegmental dynamics to interpret intralimb coordination will be discussed as they have shown invariant features during multi-segment movement. Third, studies that discovered and demonstrated a linear relationship between joint torques during movement, a relationship coined "linear synergy,” are evaluated as a consistent pattern in motor control strategy. Fourth, research that analyzed the intersegmental dynamics of movements made by neurologicallydeficient individuals (e.g., cerebellar ataxia and Down Syndrome) are presented, as they reveal underlying characteristics of normal, unconstrained movement. Fifth, research that explains differences between dominant and non-dominant arm movement are discussed to highlight and further support the invariant features found in intralimb coordination. Lastly, there will be an evaluation of research that has altered limb inertia during the intersegmental dynamic analysis of point-to-point movement.

\section{Theories explaining intralimb coordination}

Bernstein (1967) suggested that coordinated movement is associated with reducing the degrees of freedom (DOF) used by the moving joints. More recently, Stergiou, Jensen, Bates, Scholten, \& Tzetzis (2001) studied the intralimb coordination in 
running on a level surface and over obstacles, and defined coordination as the process of organizing DOFs in time and in a sequence necessary to produce a functional movement pattern. During a simple task such as reaching from point to point, the shoulder joint exhibits three DOF and the elbow exhibits two DOF; this represents a movement that can have several different trajectory paths.

In recent decades, researchers have explored how the CNS organizes movement in order to reduce these degrees of freedom for smooth, coordinated motor tasks. Two categories of theories that attempt to explain single- and multi-joint movement were generalized by Almeida et al. (2000) as 1) extrinsic, where the kinematics of the movement (e.g., velocity and trajectory) are invariant features of movement planning; and 2) intrinsic, where the CNS creates an internal representation of the movement by regulating joint torque production across various movement parameters.

One popular extrinsic motor control strategy is the equilibrium-point theory, which explains single-joint movement patterns and models a joint's musculoskeletal characteristics as a mass-spring system (Schmidt \& McGown, 1980). The equilibrium state of a joint is a static compromise between the agonist and antagonistic muscles’ viscoelastic and contractile properties. Muscle torque is generated and results in movement when this equilibrium point changes from one spatial point to another during pre-movement planning. However, the equilibrium point theory fails when trying to explain multi-joint movement (Schmidt \& Lee, 2005).

A large subset of extrinsic motor control suggests an optimal movement trajectory is produced by the joints and is governed solely by kinematics. For multi-joint movement of the upper extremity, two definitive characteristics of motor control have 
resulted when examining the kinematics of reaching: 1) the trajectory of the hand is generally a straight line, and 2) the tangential velocity profile of the hand is uni-modal and bell-shaped (Abend, Bizzi \& Morazzo, 1982). This trajectory-based theory has been supported in subsequent studies and represented by theories like the minimum hand jerk and the minimum angle jerk criterion (Schmidt \& Lee, 2005). These are cost-effective strategies that show the path taken by the hand during a reach movement closely match the trajectories generated by mathematical optimization functions of hand position and joint angle. These theories imply that the motor programs produced are somehow constrained by these mathematical representations, but they do not explain how the CNS tries to generate them.

Similar to the extrinsic optimization theories are intrinsic optimization strategies such as the minimum torque and minimum torque change criterions. Wada, Kaneko, Osu, and Kawato (2001) showed that of the four optimization criterion just discussed, the minimum torque change theory most closely matched experimental hand trajectories. Optimization theories such as the minimum torque and minimum torque change criterion have been used in areas including robotic arm manipulation due to their energy-efficient hypotheses. However, these optimization techniques do not explain motor control strategies at the muscular or neural level.

In most intrinsic motor control theories, researchers believe that the invariant kinematic characteristics of the reaching movement such as a straight line trajectory and uni-modal velocity profiles are the result of complex feedforward control over torque generation. To analyze the torques generated, the kinematics had to first be translated into kinetics through the process of inverse dynamics. Hollerbach and Flash (1982) 
authored one of the first studies that utilized this method to examine arm movement. They suggested that the difference between single-joint and multi-joint coordination is the existence of interaction torques. These torques were the result of movement by adjacent limbs and were found to be the key to unlocking the biomechanical constraints posed by Bernstein in his explanation of coordination development (Bernstein, 1967). Subsequent studies consistently incorporated inverse dynamics to investigate the intrinsic control of multi-segment coordination.

\section{$\underline{\text { Analysis of intralimb coordination using intersegmental dynamics }}$}

Schneider et al. (1989) studied the differences in coordination based on movement velocity during a sagittal plane, reversal, pointing movement. The researchers acknowledged the presence of several different torques acting simultaneously under the CNS' control. They suggested that kinematics alone could not explain the hand trajectory produced by any combination of torque patterns that are influenced by changing muscle and mechanical characteristics of the moving limbs. To evaluate the underlying kinetics involved with the movement, individual torque components were identified (e.g., net, muscular, gravitational, and interaction torques) and compared during the slowest and fastest movement trials. It was shown that during the fastest trials, control of the lower arm was influenced by the presence of large interaction torques at the elbow. These interaction torques, hinted at by Bernstein (1967) and highlighted by Hollerbach and Flash (1982) as key variables during multi-segment movement, were generated due to intersegmental effects from the upper arm to the lower arm, caused the elbow to extend, and were shown to be large contributors to net elbow torque. Elbow muscle torque acted as a counterbalance to the interaction torque and mediated the 
positioning of the distal limb during the reversal phase. Also, with practice, participants achieved faster movement times and more phasic muscle activity in contrast to the tonic and co-contraction periods of muscle activation during slow movement.

In a subsequent study of intersegmental dynamics, Schneider, Zernicke, Ulrich, Jensen, and Thelen (1990) revealed the influence of interaction torques during the kicking motion produced by supine infants. The researchers predicted the muscle torques generated by contractions would complement the passive interaction torques created during multi-segment movement. By studying movement in three-month old infants, the researchers demonstrated how these torques are generated without the benefits of years of practice in producing smooth movement. The results of the analysis yielded a similar relationship between the different joint torques during a rapid kicking motion as in the reaching movement. When muscle torque worked in the same direction as the passive interaction torques, less muscle torque was needed; when the interaction torque worked in the opposite direction as the muscle torque, more muscle torque was required during the motion. These were simple yet evident relationships between the torques at each joint that governed the levels of muscle activation generated by the CNS. Also, Schneider et al. further identified the differences in the relationship between the interaction and motion-dependent torques due to the direction of the end effector (i.e., the foot). Following a strong impulse of the proximal hip joint, distal control of muscles spanning the knee joint was manipulated to produce a smooth trajectory.

Through the study of intersegmental dynamics and electromyography (EMG) during reaching, Gribble and Ostry (1999) further demonstrated the importance and control of the interaction torques and suggested that the mechanisms of kinematic 
invariance are not determined by kinematics alone. During single-joint movement (e.g., at the elbow), EMG analysis showed anticipatory firing of the muscles spanning the stationary joint to counter the interaction torques before movement occurred at the nonstationary joint (e.g., the shoulder). As a result, elbow flexion caused shoulder extension due to these interaction torques, and anticipatory control of shoulder muscles was shown to be part of the programmed movement. In multi-joint movement, the amplitude of interaction torques was shown to vary with movement direction. Muscle activity was greater in the joints if they were moving in the same direction (flexion-flexion or extension-extension) than when the shoulder and elbow were acting in opposite directions; this demonstrated relationships that the CNS could utilize in producing coordinated movement.

The previously discussed studies evaluated whether intrinsic control of upper extremity movement exists. Through the segregation of individual torques acting at a joint, the researchers found apparent features to examine during a reaching movement, such as the influence of interaction torque on muscular control.

\section{Linear Synergy}

In the study of coordinated movement, Gottlieb et al. (1996a) studied intersegmental dynamics to determine a relationship between shoulder and elbow torques during sagittal plane motion. The researchers demonstrated that during unconstrained movement of the shoulder and elbow, the net torques produced at the joints were proportionally related over the course of the movement and represented by the following equation:

$$
\text { Torque }_{\text {shoulder }}=\mathrm{K}_{\mathrm{d}} \bullet \text { Torque }_{\text {elbow }}
$$


The coefficient $K_{d}$, a scaling factor, was shown to change with movement direction but not with movement speed. This synchronization of the joint torques produced during horizontal planar movement represented “coordinative rules” (Bernstein, 1967) that the CNS used to reduce the degrees of freedom and generate coordinated movement. Gottlieb et al. (1996a) tested this relationship with a number of different movement parameter changes that were repeated in subsequent studies. The linearity held during fast, unconstrained movement where the participants were told simply to move the hand from point to point in different angular directions. However, when the participant was asked to move with a designated trajectory (i.e., the hand path was constrained) such as in a looping underhand motion or as if drawing a gun from a holster, the torque linearities were not present. The researchers suggested that the CNS was able to modify the linear synergy rule when necessary and the rule did not exist due to biomechanical constraints.

In a follow-up study, Gottlieb, Song, Hong, Almeida, and Corcos (1996b) tested the application of the torque linearity rule, later termed "linear synergy” (Gottlieb et al., 1997), by varying joint flexion and extension and with different weights attached to the wrist. The addition of mass was hypothesized to alter the existing inertial characteristics of the joint system and therefore alter the motor program governed by the linear synergy rule. Despite the different experimental conditions, the authors showed a consistent scaling of the torques produced at the shoulder and elbow ( $\mathrm{K}_{\mathrm{d}}$ was approximately two) with distinct, biphasic torque patterns. Furthermore, it was shown that the role of the interaction torque term designated the values of the scaling constant $\mathrm{K}_{\mathrm{d}}$ : overhand throws would produce positive values, and a basketball shot would produce negative values. 
Zaal, Daigle, Gottlieb, and Thelen (1999) investigated the correlation between the linear synergy rule and a smooth trajectory produced during infant reaching to see if the linearity principle would increase in strength as the infants learned to reach with a smoother trajectory. While coordination was shown to be poor at the onset of the testing with three-week infants, the linearity principle was evident and the correlation coefficients increased through the infants' first year. It was concluded that the principle of linear synergy was not learned during musculoskeletal development as a means to create a smooth reaching trajectory, and that the development of timing constraints on the torque patterns are more likely responsible for the straightness of the hand path and its bell-shaped velocity profile. Though the study only analyzed the movement of four infants, the authors suggested from the study's findings that the linear synergy principle between joint torques is an inherent solution of the CNS to reduce the DOF problem.

Gottlieb et al. (1997) further investigated the role of movement direction in testing the linear synergy principle. Due to the changing magnitudes of flexion and extension for varying start and finish reaching points, as well as the role of gravitational joint torque, the authors wanted to find if any systematic variations in the linear synergy principle existed. In nearly all directions, the two joints demonstrated a linear synergy and biphasic torque activity (e.g., peaks in flexion and extension). The researchers discussed potential reasons for deviations from the linear synergy principle such as different muscle contraction rates, force production changes due to muscle length and shortening, reflex control, and the changing role of passive torque generation due to viscoelastic tissues across a joint. However, while none of these possibilities were supported by this study, they highlighted further aspects of coordination research. The 
authors further demonstrated the existence of the simplification of a complex motor pattern and suggested it as an optimization scheme to generate coordinated movement.

Previously discussed studies have demonstrated that the CNS utilizes a torque linearity principle when generating point-to-point movement by more than one joint and is generally true across most movement directions. The principle of linear synergy between joint torques across the motion also generates an apparent biphasic torque pattern that is consistent during planar arm movements. While the invariant kinematic features of the reach movement is not necessarily a result of linear synergy at the two joints, the torque relationship was shown to be an invariant kinetic feature during unrestrained reaching and is later demonstrated in similar studies involving intralimb coordination. Intralimb coordination in individuals with pathologies

Sainburg, Ghilardi, Poizner, and Ghez, (1995) examined the role of proprioception in intralimb control of a horizontal plane, two-joint reversal movement. The researchers' kinematic control of elbow movement isolated the changes in interaction torque that arose when moving the hand in different directions. Patients with large-fiber sensory neuropathy made bigger errors in movement direction at the reversal phase of the movement than the normal participants. The deviations were the result of reduced interjoint timing during reversals, particularly due to the lack of positional feedback. Nearly all (86\%-91\%) of the variance in elbow angular acceleration was due to peak interaction torque, a finding that repeated the importance of controlling this torque for coordinated movement. During this reversal phase, participants without proprioceptive feedback were unable to adjust the timing and onset of peak muscle 
torques to offset the effects of the elbow interaction torques, and this resulted in premature elbow flexion and erroneous hand trajectory curvatures. This study also demonstrated the influence of movement direction on whether interaction torques augmented or opposed elbow movement. Sainburg et al. (1995) demonstrated the important role of proprioception in the regulation of intersegmental dynamics, and that the programming of the motor pattern includes a feedforward loop. Also, since Sainburg et al. constrained the hand path, the muscular torques were coordinated to fit the kinematic requirements and shown to be a factor in motor control programming.

Topka, H., Konczak, J., Schneider, K., Boose, A., and Dichgans, J. (1998) compared the intersegmental dynamics of reaching at different speeds between patients with cerebellar ataxia and normal individuals. The torque profiles showed an abnormal influence of dynamic interaction torque in patients. The researchers showed that an impairment of cerebellar function is most evident in fast movements, where the lack of generating sufficient muscular forces significantly contributed to the inability to counter arising interaction torques. The gravity and interaction torques vary their influence on net torque with speed. For example, in slow movements, muscular torque is generated mostly to counteract gravity effects. In fast movements, muscular torque is used to initiate large joint acceleration then to counter arising interaction torque that is greater than gravitational effects. Therefore, patients have insufficient compensation of dynamic interaction torque, causing excessive elbow extension during the reach. Muscular torque production at the shoulder and elbow were synchronized, in that peak flexion and extension torques occurred simultaneously at both joints in patients and normal individuals. This study showed that an internal representation of the movement based on 
limb dynamics is used to generate appropriate amplitudes and timing of muscular torques. Deficiencies in this representation, as demonstrated by patients with cerebellar dysfunction, prevent accurate feedforward control of limb dynamics. In normal subjects the gravitational torque acted with the interaction torque and against generalized muscle torque. Furthermore, the analysis of the different joint torques confirmed that the principle of linear synergy was upheld during the movement.

Almeida et al. (2000) examined the linear synergy rule during horizontal plane, maximum speed, target-oriented reversal movements by individuals with Down Syndrome (DS). The deficiencies in muscular timing associated with these individuals were hypothesized to alter the CNS’ response to interaction torques that occurred during the multi-segment movement. Resultant similarities in hand path trajectories between DS patients and normal individuals suggested that, in contrast to individuals with proprioceptive deficits, the neurological basis of DS does not play a role in altering the internally-produced model of the movement. While the results showed different reaction times (300 milliseconds for individuals with DS compared to 150 milliseconds for normal individuals), the authors found no distinct pattern in interaction torque between patients and normal individuals.

The results from Almeida et al. (2000) failed to identify significant patterns of interaction torque that differentiated between neurologically normal individuals and those with DS. However, in contrast to their hypotheses, patients were able to make sharp reversals in hand trajectory and they did not show periods of delay at the reversal region or large deviations in the return path. Movement times and torque amplitudes were lower in patients, but the differences were not as distinct as in those with cerebellar ataxia. In 
this case, the linear synergy rule was weakly held. The researchers reported no significant deviations from this rule, but found it did not depend on movement speed. Furthermore, individuals with DS showed more fluctuations in muscular torque which may confirm that the ratio of torque varies with movement direction and torque magnitude.

Taken together, these studies (Sainburg et al., 1995; Topka et al., 1998; Almeida et al., 2000) demonstrate the importance in feedforward control of the passive, interaction torques that arise at the joints during multi-segment movement. For example, Down Syndrome patients did not show differences in the scaling of shoulder and elbow torques nor did they show any discernible differences in kinematic or kinetic features of horizontal plane movement. Therefore, in the hierarchy of motor control that exists in generating the reaching movement, the findings of these studies suggest that all participants employ movement strategies like the linear synergy rule.

\section{$\underline{\text { Hand dominance }}$}

Sainburg and Kalakanis (2000) investigated the differences in kinematics and kinetics between dominant and non-dominant limb movement during unconstrained point-to-point movement. An analysis of intersegmental dynamics demonstrated that with greater shoulder joint movement, larger elbow interaction torques were developed and that these interaction torques contributed a higher percentage of the elbow net torque. In other words, with larger range-of-motion (ROM) movement of the shoulder at a fast speed, less elbow muscle force was used to generate elbow movement. The passive interaction torque generated at the elbow was utilized and contributed more towards the net elbow torque with larger shoulder joint excursions. Elbow interaction torque 
contribution towards net elbow torque was approximately 20 percent more at the right elbow than the left elbow regardless of direction. This shows that the dominant arm was more efficient in using the interaction torque to generate elbow movement and described a marked difference in intralimb coordination between dominant and non-dominant limbs. Differences in path trajectories were noted (e.g., the non-dominant arm had more rounded deviations and the dominant arm showed near straight paths to target), including larger amounts of elbow extension for a given amount of shoulder flexion in the nondominant arm. The differences described were thought to be an advantage in performing the motor pattern due to the reduced muscle torque requirements as well as the more accurate hand paths taken by the dominant arm. The study also noted the independence of hand path shape from interaction torque of the dominant arm, whereas hand path shape of the non-dominant arm was heavily dependent on interaction torque. The researchers thought this result was due to less accurate predictions of the interactions. The findings of Sainburg and Kalakanis (2000) not only highlighted the importance of controlling interaction torque but also demonstrated that the CNS utilizes arising interaction torque in its favor during movement planning to determine efficient muscle torque generation.

Considering the findings of Sainburg and Kalakanis (2000), a subsequent study examined whether the difference in torque efficiency was due to intrinsic planning features or a secondary result of kinematics (Bageistro \& Sainburg, 2002). They controlled for most variables in horizontal, rapid speed pointing, and by controlling target direction were able to isolate interaction torques generated at the elbow. EMG analysis proved that the torques produced by the dominant arm were significantly less than that of the non-dominant arm to produce movements with similar speed, movement time, and 
hand path trajectories. Despite small differences in trajectories (e.g., the dominant hand showed more of a straight line and non-dominant hand showed curved paths), the authors were convinced that the dominant arm utilizes a torque-efficient strategy whereas the non-dominant arm does not.

Studies that alter inertial properties during the reaching movement

Sainburg et al. (1999) analyzed torque profiles of the reaching movement during three trial sets with different inertial characteristics: 1) medially-placed, mass-adapted trials; 2) surprise trials where the mass was placed laterally without participant knowledge; and 3) in laterally placed, mass-adapted trials. In the lateral mass trials, deviations in hand paths shown by the surprise trials were reduced and trajectories converged towards mass-adapted trajectories. The findings showed that following the participant's learning of the mass-adapted movements, an anticipatory response was found in the motor control of movement when removing the mass, which was a new movement goal. From a motor control standpoint, the authors showed that an intrinsic feedforward system does exist which adapts to novel loading conditions. For example, when currently learned internal representations of the intersegmental dynamics involved in a movement are suddenly perturbed (e.g., limb inertial alteration), the strategy that involved coupling joint movement and timing between the shoulder and elbow was not effective in countering the increase in interaction torques and resulted in large deviations from straight-line trajectories.

Sainburg and his co-workers demonstrated that the CNS adapted to a medially placed mass and corrected trajectory deviations to achieve a straight line reversal. During movement initiation, the shoulder flexed, which caused an interaction torque that 
extended the elbow. Under no-load conditions, the elbow flexors would have countered this and the path would have remained straight. However, the elbow muscle torque could not counter the surprise increase in inertia, which resulted in large lateral deviations and a rounded curve. During the return movement, the elbow extensors initiated after the elbow interaction torque caused elbow flexion, which caused an initial medial deviation during the return path. Nonetheless, this deviation was corrected and the hand returned near the starting position through elbow extensor control. The authors stated that the comparison of pre-loading conditions to the surprise loading showed initial similarities in torque generation: this suggested anticipatory responses in the motor control strategy were followed by feedback-based responses in torque output to model the predetermined kinetic strategy. At the end of the movement the authors suggested that a postural mechanism that was less related to the intersegmental dynamics of the movement returned the deviated trajectory back to its intended endpoint goal.

In a later study, Sainburg (2002) examined hand path trajectory and intersegmental dynamics following the addition of a one kg mass eccentrically displaced from the long axis of the forearm. The generated torques showed a linear synergy between the shoulder and elbow in approximately a two-to-one ratio, respectively. A comparison of dominant and non-dominant arm performance following the removal of the mass showed that hand path deviations and the time course of adaptation were similar to performances before adding the mass. Practice effects resulted in larger improvements in dominant arm performance towards that of pre-mass conditions in comparison to the non-dominant arm. With regards to the torques generated, the baseline conditions were in agreement with the Sainburg et al. (1999) study in that less shoulder torque was used 
because the dominant arm utilized the elbow interaction torques. The non-dominant arm's net shoulder torque was comprised mostly of shoulder muscle torque as it counteracted higher elbow muscle torques. In contrast, for dominant arm movements, elbow muscle torque contributed to the shoulder net torque by acting in the same direction. The authors noted that the differences in torque production may be due to differences in maximal torque output by the dominant versus the non-dominant limbs. However, analysis of the quantitative data showed that the mean-squared torque generated by the non-dominant limb was greater than that of the dominant limb for similar movement velocities.

As the results of the Sainburg study (2002) indicate, CNS control of torque generation results in trajectories similar to those of mass-adapted conditions and further exhibits a strategy to optimize torque profiles and efficiency. From a motor control standpoint, learning effects that occurred several trials following the addition of the mass and after transfer of the mass to the other side of the arm, created progressively linear handpaths and "after effects," deviations in the initial and return handpaths that were mirror reflections of the adapted performance. In comparing dominant to non-dominant arm performance, the dominant arm showed more complete adaptations.

Similar works also supported the CNS' ability to adapt intralimb coordination in novel loading conditions towards coordination in non-loaded conditions. Through the analysis of elbow and shoulder torques during a point-to-point reversal movement, Debicki and Gribble (2004) displayed that the correction of positional errors were primarily due to changes in elbow muscle torque. They suggested the changes were made by incorporating visual feedback error into the feedforward loop while 
implementing the preprogrammed movement. While changes in shoulder torque were evident, these were in the same direction and proportional to the changes in elbow muscle torque. These findings suggested that the CNS attempts to maintain a coupled relationship between shoulder and elbow torques generated during unconstrained movement.

Summary

The research discussed in this review represents a comprehensive undertaking to determine the essential features of intralimb coordination of the arm during a discrete motor task such as reaching. While extrinsic, kinematic analysis of the reaching movement show invariant characteristics such as a bell-shaped velocity profile and a linear hand path trajectory, the study of intersegmental dynamics has demonstrated that an internal representation of the movement exists. These intrinsic motor control strategies relate individual torques during the movement and highlight the significance of the passive interaction torque arising between limb segments. Through the control of movement parameters and subsequent torque analyses, researchers like Gottlieb demonstrated a linear synergy exists between shoulder and elbow muscle torques during a reaching movement, and this finding was supported by other intralimb coordination studies. Studies of individuals with movement pathologies such as cerebellar ataxia (Topka et al., 1998), and Down Syndrome (Almeida et al., 2000) highlighted invariant features in movement control such as the counterbalancing of interaction torque and Gottlieb’s linear synergy principle. Hand dominance studies explained differences in motor control between dominant and non-dominant limbs, such as the counterbalancing of elbow interaction torque with muscle torque, and the dominant arm's efficiency to 
reduce torque output through temporal optimization. Finally, studies that introduced perturbations to the inertial parameters of the reaching movement showed that with practice, intralimb coordination converged towards pre-perturbation torque patterns and faster movement times. This demonstrated a motor control strategy to maintain torque output efficiency and also maintained a linear relationship between shoulder and elbow torque production. Thus, the previously review studies highlight the current understanding of intralimb coordination and characteristics of multi-segment movement. By analyzing joint torques using intersegmental dynamics, an internal representation of motor planning was demonstrated in the identification of specific torque patterns. While some studies evaluated these torque patterns after altering limb inertia, none added inertia solely to the upper arm. Most of were also performed in the horizontal plane. Therefore, the analysis of joint torques and the CNS' response to altered arm inertia in the sagittal plane may provide additional understanding of intrinsic motor control strategies. 


\section{CHAPTER 3}

\section{METHODS AND PROCEDURES}

The purpose of this study was to evaluate the effects of altered upper limb inertia on the coordination between elbow and shoulder torque production during a reversal pointing movement. This study was a pretest-posttest experimental design in which the kinematic and kinetic variables of the participants (i.e., dependent variables) were analyzed with and without added weight (i.e., independent variable) to the upper arm. This chapter describes the participants, test procedures, and statistical analyses used in this study.

\section{$\underline{\text { Participants }}$}

Ten right-hand dominant males and females aged 27 to 39 years (mean age $=33.4$ years, standard deviation $=4.3$ ) residing in San Luis Obispo County volunteered to participate in this study from August to September 2008. Each participant gave informed consent, and the study's design and experimental procedures were approved by the Cal Poly Human Subjects Committee.

\section{$\underline{\text { Procedures }}$}

Upon the arrival of each participant to the lab, the researcher gave the participant a brief overview of the equipment, safety concerns, and general procedure. Each participant reviewed and signed an informed consent form as well as a brief sports history survey for a subsequent analysis of participant homogeneity (see Appendix A for a copy of the form and survey). Next the participant was asked to sit in a chair positioned in front of the target apparatus. The chair was located approximately $457 \mathrm{~mm}$ from the ground. Two foam targets were distanced 508mm vertically apart, with a transverse- 
oriented acrylic barrier 229mm x 305mm x 3mm between the targets around which the hand had to circumvent. The barrier was located $457 \mathrm{~mm}$ from the participant's abdomen (Schneider et al., 1989). Figure 1 shows the experimental setup and the general hand path performed in each trial: start from Target 1, around the barrier to Target 2, and back down around the barrier to Target 1.

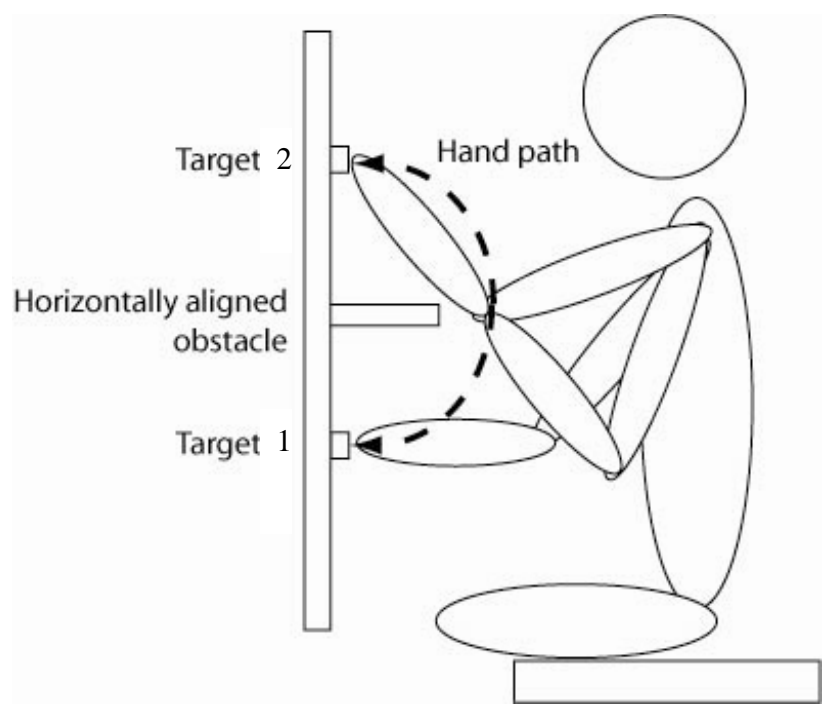

Figure 1. Experimental Setup of Participant, Barrier, and Target Locations.

Next the researcher placed reflective markers on the shoulder, elbow, and wrist using double-sided tape. The researcher briefly demonstrated the movement and instructed the participant to move as fast as possible in the sagittal plane without warm-up or practice trials. The researched then turned on the JVC video camera to start recording the trial. The camera was mounted to a stationary tripod and placed approximately 3 meters from the participant; it recorded the trials at $60 \mathrm{~Hz}$.

To initiate the movement, the researcher said to the participant, "Ready, set, go!” after which the participant performed one trial. Based on a pre-experiment pilot study and past research (Schneider et al., 1989; Gottlieb et al., 1996b) each participant 
completed 10 trials in a weighted condition and 10 trials in a non-weighted condition.

The participant was allowed to rest for five seconds between each trial, and a three minute rest was given between the two sets of trials. The order of the two sets of trials was randomly assigned to each participant. The weighted condition consisted of a $2.2 \mathrm{~kg}$ weight wrapped around the middle of the right upper arm and secured by a soft wrap bandage without discomfort to the participant and without hindering the movement. The unweighted condition consisted of no weight attached to the arm.

Participants were instructed to move in the sagittal plane, but no external constraints were used to ensure sagittal plane movement. However, the researcher stood behind the participant and visually checked for any large deviations from the sagittal plane. If a participant made a large deviation, did not finish a trial, or other errors occurred (e.g., participant hit the barrier, missed a target, or moved outside of the movement plane), that trial was not used in the data analysis. The participant continued until 10 successful trials in each condition were completed.

The video trials were processed by Vicon’s Motus software (Vicon, Los Angeles, CA) to create kinematic data. This data were further processed using inverse dynamic equations with Microsoft Excel 2003 (Microsoft, San Jose, CA) to result in joint torque values produced throughout the movement.

\section{$\underline{\text { Data Computations }}$}

Kinematic data of the wrist, elbow, and shoulder joints were generated based on angular position orientation from Gottlieb et al. (1996a \& 1996b) and displayed in Figure 2. 


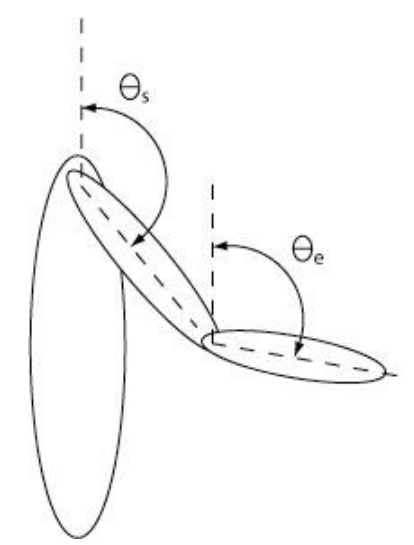

Figure 2. Orientation of the Shoulder and Elbow Angles for Kinematic Calculations.

The angle of the elbow with respect to the upper arm is given by $\phi=\theta_{e}-\theta_{s}$. Joint angles, velocities, and accelerations were combined with participant height and weight to compute shoulder and elbow torque using inverse dynamics equations. First, limb lengths and center of mass locations for each participant were used to compute principal moments of inertia (Winter, 2004). When the $2.2 \mathrm{~kg}$ weight is added to the elbow, the weight is treated as a point mass in the calculations. This anthropometric information was then inserted into previously defined equations (Gottlieb et al., 1996a \& 1996b) that produced joint muscular torque data (see Appendix C for these equations).

Data collection focused on a movement period defined by temporal landmarks similar to Schneider et al. (1989), and Almeida et al. (2000): it began when the hand rounded the barrier (transition from elbow flexion to extension) towards Target 2, continued through reversal (transition from elbow extension to flexion) at Target 2, and ended when the hand rounded the barrier towards Target 1 (transition from elbow flexion to extension). Movement time was derived by taking the number of frames within this period and multiplying by the inverse of the video acquisition rate, or 1/60 seconds/frame. 
Of the 10 trials for each participant in each condition, the three fastest trials (i.e., lowest movement period) were used to calculate mean shoulder torque (Ts), mean elbow torque (Te), and mean movement time (t). The fastest movement trials were used because they generated the highest joint interaction torques (Gribble \& Ostry, 1999). Subsequently, the CNS reacts to these interaction torques and incorporates them into the resultant kinetic strategy for hitting each target (Hollerbach \& Flash, 1982; Gribble \& Ostry, 1999).

\section{Data Analysis}

Mean shoulder torque, mean elbow torque, and mean movement time were independently compared between the weighted and non-weighted conditions using dependent t-tests to determine the effects of the added upper arm weight. For all comparisons, a level of $p=0.05$ was used to determine statistical significance. 


\section{CHAPTER 4}

\section{RESULTS AND DISCUSSION}

To determine the effects of added inertia to the dominant upper arm on the coordination between elbow and shoulder torque production during a reversal reaching movement, kinetic motion data of 10 participants in weighted and non-weighted conditions were analyzed. This chapter presents the results of the analysis and discusses their significance.

\section{$\underline{\text { Results }}$}

Figure 3 depicts a typical profile of muscular shoulder and elbow torques normalized with joint angular position over the entire reversal movement. The movement exhibited zero-torque crossings near points of flexion-extension transitions similar to other reversal movement studies (Sainburg et al., 1999). The shaded area depicts the movement period over which data collection was defined: upward around the barrier towards Target 2, reversal at Target 2, then downward around the barrier towards Target 1. Peak values of shoulder and elbow torques occurred at the reversal point of the movement at Target 2; this is consistent with other findings that at reversal regions, joint muscular torque reacts to the large increase in interaction torque between adjacent limbs (Schneider et al., 1989). For all participants, elbow torque values were relatively small in comparison to shoulder torque. These torque profiles were analyzed for consistency within each participant's data. With one participant, one of the three trials used to determine mean values was excluded because there were unexplained aberrations in shoulder torque at the reversal point. 


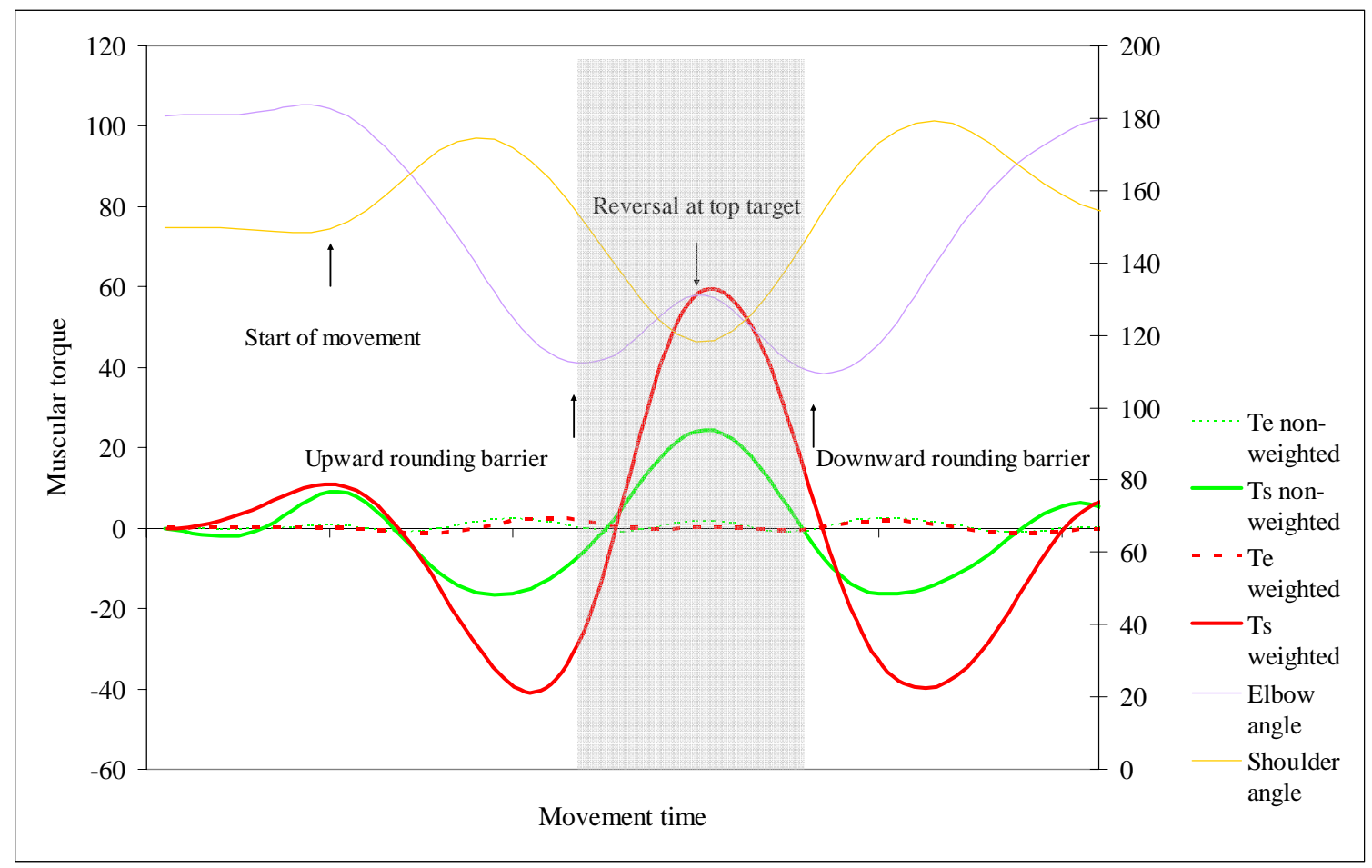

Figure 3: Typical Joint Angle and Torque Profiles Normalized Across Each Weight Condition For One Participant.

Mean muscular shoulder torque (Ts), mean muscular elbow torque (Te), and mean movement time (t) for the weighted and non-weighted conditions are shown in Table 1 (see Appendices $\mathbf{D}$ and $\mathbf{E}$ for individual participant data).

Table 1: Mean Torque and Time Data (Mean \pm Standard Deviation) Across Weight Condition (n=9).

\begin{tabular}{|c|c|c|}
\hline & Non-weighted & Weighted \\
\hline $\begin{array}{c}\text { Shoulder torque, Ts } \\
(\mathrm{N}-\mathrm{m})\end{array}$ & $16.16 \pm 7.51^{*}$ & $41.42 \pm 25.07^{*}$ \\
\hline $\begin{array}{c}\text { Elbow torque, Te } \\
\text { (N-m) }\end{array}$ & $0.99 \pm 0.76$ & $0.72 \pm 0.40$ \\
\hline $\begin{array}{c}\text { Movement time, t } \\
\text { (seconds) }\end{array}$ & $0.27 \pm 0.07^{*}$ & $0.29 \pm 0.08^{*}$ \\
\hline
\end{tabular}

Note: Statistical significance $(*)$ between groups at $p<0.05$. 
Movement time was significantly slower $(p>0.05)$ with added weight on the upper arm. Mean peak muscular shoulder torque was significantly larger $(p<0.05)$ with the added weight. Mean peak muscular elbow torque was not significantly different $(p=0.1074)$ between the weighted and non-weighted conditions (see Appendix E for a summary table of the statistical results). However, for all except two participants, the elbow torque was less with the added weight. 


\section{CHAPTER 5}

\section{SUMMARY, CONCLUSIONS, AND RECOMMENDATIONS}

The purpose of this study was to determine the effects of added inertia on the coordination between elbow and shoulder torque production during a sagittal plane, reversal reaching movement.

\section{Summary}

Five males and five females aged 27 to 39 years (mean age $=33.4, \mathrm{SD}=4.3$ ) each performed ten trials with a $2.2 \mathrm{~kg}$ weight attached to their upper, dominant right arm and ten trials without the weight. Each trial consisted of a reversal pointing movement in the sagittal plane around a small barrier as fast as possible. These video-taped trials were processed into kinematic motion data then converted into torques using anthropometric statistics and inverse dynamic equations. As a result of these computations, mean peak shoulder torque, mean peak elbow torque, and mean movement time were analyzed during the reversal region of the movement. Data analysis of these dependent measures showed mean movement time was significantly larger (i.e., slower) with the added weight than without it $(p<0.05)$. Mean peak shoulder torque was significantly larger with the added weight than without it $(p<0.05)$. Mean peak elbow torque was not significantly different with the added weight than without it $(p=0.1074)$.

\section{$\underline{\text { Conclusions }}$}

All except one participant moved slower during the reversal region with the added inertia than without it. Slower movement times were expected for the CNS to overcome the additional inertia while accelerating and decelerating within the movement period. Though not all participants exhibited an increase in movement time with the weight, 
future studies may determine whether the CNS' adaptation to the increased inertia is affected by practice.

Trials with the added weight led to expected increases in shoulder torque: for some participants a threefold increase was measured. The increase can be attributed to the shoulder's role as the primary mover of the multi-segment system for generating large, gross movements (Schmidt \& Lee, 2005). Thus, as the hand moved around the barrier and transitioned from shoulder flexion to extension, shoulder torque production was elevated to compensate for the increased inertia and accelerate the hand towards Target 2 .

In trials with the added weight, the increase in shoulder torque production was accompanied by small decreases in elbow torque. While this reduction was not found to be significantly different than without the weight, all except two participants’ mean elbow torque values were lower with the weight. This is consistent with previous research (Schneider et al., 1989; Hollerbach \& Flash, 1982) in which large torque values at the shoulder resulted in large interaction torques arising at the elbow.

Depending on the movement goal and target direction, elbow muscular torque was shown to either counterbalance or combine with the elbow interaction torque to accomplish the movement goal. For the latter situation, less elbow muscular torque was needed to achieve the required net torque for the movement. This explains the relatively smaller torque generated at the elbow compared to the shoulder. In other words, due to the arising interaction torque at the elbow, the elbow did not need to produce a great amount of muscular torque to achieve the movement goal. Similarly, the increase in shoulder torque as a response to added inertia resulted in a larger elbow interaction 
torque, thus even less muscular torque required to achieve the movement goal.

Furthermore, because the elbow is the more distal joint in the multi-segment system, it is responsible for refining the movement in response to the gross movements of the shoulder. With more mass to move, the shoulder increased its torque production, which developed higher interaction torque at the elbow, where torque production was reduced. To verify the reduction in muscular torques presented by the computations and analysis, an electromyography (EMG) analysis of elbow joint muscles could be performed.

The results of this study showed a significant increase in shoulder torque accompanied by a trending decrease in elbow torque as a response to increased upper arm inertia. While not conclusive, this study demonstrated a coordinated response between the shoulder and elbow that is supported by previous research (Bernstein, 1967; Hollerbach \& Flash, 1982; Schneider et al., 1989; Gottlieb et al., 1996a \& 1996b; Gribble \& Ostry, 1999; Debicki \& Gribble, 2004). Some studies showed a linear relationship between shoulder and elbow torque generation during a pointing movement (Gottlieb et al., 1996a \& 1996b). However, this study did not show a linear relationship during the defined movement period, perhaps because the movement goals and parameters were not the same as previous works. A follow-up study could examine a similar reversal pointing movement in the sagittal plane without a barrier.

The decrease in elbow muscular torque during trials with the added weight was a response to increasing interaction torque at the elbow. Analysis of the interaction torque by separating its computational term within the inverse dynamic equations (Schneider et al., 1989) may give further insight into the response of shoulder and elbow torque 
generation and allow a more quantitative analysis of each torque's contribution during the movement.

Compared to those with pathological (Almeida et al., 2000; Topka et al., 1998) or proprioception deficits (Sainburg et al., 1995), normal participants were able to react to changes in interaction torques and accurately accomplish the movement goal. This study showed that more shoulder torque and less elbow torque was needed to adapt to the increased upper arm inertia and maintain movement accuracy at each target. However, the adaptation was not shown to be statistically consistent and warrants further investigations.

The results of this study further support Bernstein’s original proposals (Bernstein, 1967) that human movement is made efficiently and uses natural occurring phenomena (i.e., joint interaction torques) to facilitate multi-segment movement. Previous research (e.g., Debicki \& Gribble, 2004) has shown that the central nervous system adapts to external changes in movement planning and can accurately achieve the movement goal by altering torque output at each joint. This study showed that the CNS consistently increased shoulder torque and decreased elbow torque. This pattern in joint torque production represents another example of the CNS' ability to adapt to changes and accomplish movement goals.

\section{$\underline{\text { Recommendations }}$}

Based on this study's findings, the following recommendations are given:

1. Not all participants in this study showed a slower mean movement time in trials with the added weight. Because the participants had to learn how to move around the barrier at fast speeds, the movement period defined in this study may have 
produced variations that affected the findings. Thus, future studies that examine practice effects may determine a more representative movement period with and without the weight.

2. This study did not result in a linear relationship between elbow and shoulder torques during a reversal pointing movement as in previous research (Gottlieb et al., 1996a \& 1996b). These previous works did not incorporate a barrier (Schneider et al., 1989) as done in this study. Thus, future investigations that examine the effects of increased upper arm inertia during a movement without a barrier may show a linear synergy between the shoulder and elbow.

3. This study showed a consistent increase in shoulder torque but a non-consistent decrease in elbow torque during trials with the added weight. To more clearly determine whether elbow torque reduction is part of the CNS' planning, future studies could redefine the movement. As discussed earlier, the movement around the barrier determined the start and subsequent finish of the movement period. The ability of each participant to circumvent the barrier may have affected the coordination between shoulder and elbow. Thus, future studies can examine the sagittal plane, reversal pointing movement without the barrier. 


\section{BIBLIOGRAPHY}

Abend, W., Bizzi, E., \& Morazzo, P. (1982). Human arm trajectory formation. Brain, 105, 331-348.

Almeida, G., Corcos, D., \& Hasan, Z. (2000). Horizontal-plane arm movements with direction reversals performed by normal individuals and individuals with down syndrome. Journal of Neurophysiology, 84, 1949-1960.

Bageistro, L, \& Sainburg, R. (2002). Handedness: dominant arm advantages in control of limb dynamics. Journal of Neurophysiology, 88, 2408-2421.

Bernstein, N. (1967). The co-ordination and regulation of movements. Oxford, UK: Pergamon Press.

Crossman, E.R.F.W., \& Goodeve, P.J. (1963/1983). Feedback control of handmovements and Fitts’ law. Paper presented at the meeting of the Experimental Psychology Society, Oxford, July, 1963. The Quarterly Journal of Experimental Psychology, 1983, 35A, 251-278.

Debicki, D. \& Gribble, P. (2004). Inter-joint coupling strategy during adaptation to novel viscous loads in human arm movement. Journal of Neurophysiology, 92, 754-765.

Gottlieb, G., Song, Q., Hong, D., \& Corcos, D. (1996a). Coordinating two degrees of freedom during human arm movement: load and speed invariance of relative joint torques. Journal Of Neurophysiology, 76 (5), 3196-3206.

Gottlieb, G., Song, Q., Hong, D., Almeida, G.L., \& Corcos, D. (1996b). Coordinating movement at two joints: A principle of linear covariance. Journal of Neurophysiology, 75 (4), 1760-1764. 
Gottlieb, G.L., Song, Q., Almeida, G.L., Hong, D., \& Corcos, D. (1997). Directional Control of Planar Arm Movement. Journal of Neurophysiology, 78, 29852998.

Gribble, P. \& Ostry, P. (1999). Compensation for interaction torques during singleand multijoint limb movement. Journal of Neurophysiology, 82, 2310-2326.

Hollerbach, J. \& Flash, T. (1982). Dynamic interactions between limb segments during planar arm movement. Biological Cybernetics, 44, 67-77.

Kreighbaum, E. \& Barthels, K. (1996). Biomechanics: a qualitative approach for studying human movement, $4^{\text {th }}$ edition. Needham Heights, MA: Allyn \& Bacon.

Poulton, E.C. (1957). On prediction in skilled movements. Psychological Bulletin, 54, 467-478.

Sainburg, R. (2002). Evidence for dynamic-dominance hypothesis of handedness. Experimental Brain Research, 142, 241-258.

Sainburg, R. \& Kalakanis, D. (2000). Differences in Control of limb dynamics during dominant and nondominant arm reaching. Journal of Neurophysiology, 83, 2661-2675.

Sainburg, R., Ghez, C., \& Kalakanis, D. (1999). Intersegmental dynamics are controlled by sequential anticipatory, error correction, and postural mechanisms. Journal of Neurophysiology, 81, 1045-1056.

Sainburg, R., Ghilardi, M., Poizner, H., \& Ghez, C. (1995). Control of limb dynamics in normal subjects and patients without proprioception. Journal of Neurophysiology, 73(2), 820-835. 
Schmidt, R. \& Lee, T. (2005). Motor control and learning: a behavioral emphasis, $4^{\text {th }}$ edition. Champaign, IL: Human Kinetics.

Schmidt, R. \& McGown, C. (1980). Terminal accuracy of unexpectedly loaded rapid movements: evidence for a mass-spring mechanism in programming. Journal of Motor Behavior, 12(2), 149-161.

Schneider, K., Zernicke, R., Schmidt, R., \& Hart, T., (1989). Changes in limb dynamics during the practice of rapid arm movements. Journal of Biomechanics, 22(8/9), 805-817.

Schneider, K., Zernicke, R., Ulrich, B., Jensen, J., \& Thelen, E. (1990). Understanding movement control in infants through the analysis of limb intersegmental dynamics. Journal of Motor Behavior, 22(4), 493-520.

Stergiou, N., Jensen, J., Bates, B., Scholten, S,, \& Tzetzis, G. (2001). A dynamical systems investigation of lower extremity coordination during running over obstacles. Clinical Biomechanics, 16, 213-221.

Topka, H., Konczak, J., Schneider, K., Boose, A., \& Dichgans, J. (1998). Multijoint arm movements in cerebellar ataxia: abnormal control of movement dynamics. Experimental Brain Research, 119, 493-503.

Wada, Y., Kaneko, E., Osu, R., \& Kawato, M. (2001). Quantitative examinations for multi-joint arm trajectory planning - using a robust calculation algorithm of the minimum commanded torque change trajectory. Neural Networks, 14, 381-393.

Winter, David. (2004). Biomechanics and motor control of human movement, $3^{\text {rd }}$ ed. New York: Wiley \& Sons. 
Zaal, F., Daigle, K., Gottlieb, G., \& Thelen, E. (1999). An unlearned priniciple for controlling natural movements. Journal of Neurophysiology, 82, 255-259. 
Appendix A

Informed Consent Form 


\section{Human Subjects Research Protocol Cal Poly, San Luis Obispo INFORMED CONSENT FORM}

Title of the research: The Effects of Alterations to Limb Inertial Properties on Vertical Point-to-Point Movement of Upper Extremity

Principal Investigator: Adrian K. Hongo, Kinesiology Department, (805) 215-4147

Faculty Advisor: Dr. Robert D. Clark, Kinesiology Department, (805) 756-0285

The testing which you will be participating in is for research purposes. This form will advise you of the testing procedures and the risks involved. The purpose of the study is to add to an existing body of research in movement coordination, specifically the coordination between the shoulder and elbow joints during a reach movement. Your identity as a participant in this testing will be kept confidential at all times. For your participation, you will be offered food and drink by the Investigator.

The expected duration of your participation will be between 30-45 minutes starting from your arrival to the testing location, which is Room 153 Biomechanics Laboratory, Building 43 Kinesiology on the Cal Poly campus. When you arrive, you will be briefed of the testing procedure, which is summarized below:

- Calibration video of the setup.

- You will be assigned a user code to track your data (e.g., "M1" for male participant \#1, or "F2" for female participant \#2). If signed, this consent form will list the user code assigned to you. Record of this information is necessary in the case of any problems in the data collection and/or results that may arise which requires contacting you, or any other issue that may require identification of your participation.

- Your height and weight will be recorded.

- Placement of reflective markers with double-sided tape on your right shoulder, elbow, and wrist.

- You will sit on a backless chair facing the frame with a horizontal plastic barrier.

- Each trial consists of an unconstrained, fast-as-possible movement of your right hand around the barrier from point 1 to point 2 then back to point1.

- 10 trials will be performed with 20 seconds of rest between each trial.

- Next a 5lb. weight will be comfortably wrapped around your upper arm above your elbow.

- You will perform 10 more trials of the same movement, with 20 seconds of rest in between each trial.

- Markers will be removed, and following a rest and any questions, you'll be free to leave the testing lab.

Potential risks during your participation of this research include the following: 
- Muscular fatigue

- Injury to hand/arm during movement around the plastic barrier

- Psychological distress due to recording of your height/weight

At any point during the testing, if you feel fatigued and/or are unwilling to continue, the testing and video recording will be stopped and you will be free to leave. If you decide that you do not want your results included in the research, the data will not be used in developing any research conclusions. Any questions, report of harm, or to obtain a summary of the study's results, please contact the Principal Investigator or Advisor at the phone numbers above. You can also contact Health Services at 756-1211, or in case of an emergency, dial 911 on the phone in the Biomechanics Lab where the testing will be held.

You participation is completely voluntary and no penalty will result from your refusal to participate or stoppage during participation once initiated.

Your confidentiality will be protected. Your participation in this research will be video taped; these video tapes will be kept off campus at the Investigator's home. The computer files that reference the videos will be password protected using a password known only to the Investigator. This form will be stored by the Investigator at his home until the completion, acceptance and subsequent publication of this thesis project, upon which time all these forms will be destroyed, video tapes erased, and computer files deleted.

If you have questions regarding this study or would like to be informed of the results when the study is completed, please feel free to contact the Investigator, Adrian Hongo at 805-215-4147, or Dr. Robert Clark, the Investigator's faculty advisor, at 805-756-0285. If you have questions or concerns regarding the manner in which the study is conducted, you may contact Steve Davis, Chair of the Cal Poly Human Subjects Committee, at 7562754, sdavis@calpoly.edu, or Susan Opava, Dean of Research and Graduate Programs, at 756-1508, sopava@calpoly.edu.

If you agree to voluntarily participate in this research project as described below, please indicate your agreement by signing below. Please keep one copy of this form for your reference, and thank you for your participation in this research.

Signature of Volunteer: Date: Date: 


\section{Appendix B}

Pre-Experiment, Sports Participation Survey 


\section{Participant Survey}

\section{Participant ID\#}

Please circle each sport/activity you have participated in during the past 10 years. For each one you've participated in, please indicate

1) level of expertise and 2) approximate number of years played.

Experience Level:

(Beginner/Novice/ExpertPro)

\section{Years Played:}

(Approximate)

\section{Archery}

Darts

Soccer

Baseball

Softball

Basketball

Bowling

Golf

Water Polo

Volleyball

Swimming

Cycling

Martial Arts

Ping Pong 


\section{Appendix C}

Inverse dynamics equations used to convert kinematic data to joint torques:

\section{Elbow muscular torque $=$}

Il X d2thetae +

$$
\begin{aligned}
& \text { rl X lengf X mf X cos(thetae) } X \text { d2thetas }+ \\
& \text { rl X lenga X mf X sin(thetae) } X \text { dthetas }{ }^{\wedge} 2+ \\
& \text { rl X mf X sin(thetae) } X \text { g }
\end{aligned}
$$

\section{Shoulder muscular torque $=$}

$$
\begin{aligned}
& \text { (Iu+lenga^2 X mf) X d2thetas + } \\
& \text { (rl X lenga X ma X cos(thetae) X d2thetae + } \\
& \text { rl X lenga } X \sin \left(\text { thetae) } X \text { dthetae }^{\wedge} 2+\right. \\
& \text { ((ru X ma)+(lenga X ma)) X sin(thetas) X g + }
\end{aligned}
$$

\section{Elbow torque}

Definition of equation terms:

$$
\begin{aligned}
& \mathrm{g}=\text { gravitational constant (= } 0 \text { for isolating muscular torque) } \\
& \mathrm{rl}=\text { distance from elbow joint to lower arm center of mass } \\
& \mathrm{ru}=\text { distance from shoulder joint to upper arm center of mass } \\
& \text { lengf = forearm length (=0.43 X body height) }{ }^{\text {Winter }} \\
& \text { lenga = upper arm length (=0.436 X body height) }{ }^{\text {Winter }} \\
& \text { mf = mass of lower arm (=0.051 X } 0.333 \mathrm{X} \text { participant mass })^{\text {Winter }} \\
& \text { ma = mass of upper arm (=0.0510.549 X participant mass })^{\text {Winter }} \\
& \text { thetaf = elbow angle with vertical } \\
& \text { dthetaf = elbow angular velocity }
\end{aligned}
$$


d2thetaf = elbow angular acceleration

thetaa $=$ shoulder angle with vertical

dthetaa $=$ shoulder angular velocity

d2thetaa $=$ shoulder angular acceleration

thetae $=$ elbow angle (thetaf - theta)

thetae $=$ elbow angle with vertical

dthetae $=$ elbow angular velocity

d2thetae $=$ elbow angular acceleration

$\mathrm{Il}=$ Moment of inertia of the lower $\operatorname{arm}\left(=\mathrm{rl}^{\wedge} 2\right)$

$\mathrm{Iu}=$ Moment of inertia of the upper arm (unweighted $=\mathrm{ru}^{\wedge} 2$, weighted $=\mathrm{Iu}+\mathrm{ma} \mathrm{X} \mathrm{ru} \wedge 2$ ) 


\section{Appendix D}

Mean Values of Each Participant's Data:

\begin{tabular}{|c|c|c|c|c|c|c|c|c|c|}
\hline $\begin{array}{c}\text { Participant } \\
\text { ID }\end{array}$ & $\begin{array}{c}\text { Age } \\
\text { (Years) }\end{array}$ & $\begin{array}{c}\text { no } \\
\text { weight }\end{array}$ & weight & $\begin{array}{c}\text { no } \\
\text { weight }\end{array}$ & weight & $\begin{array}{c}\text { no } \\
\text { weight }\end{array}$ & weight & $\begin{array}{c}\text { no } \\
\text { weight }\end{array}$ & weight \\
\hline m5 & 39 & 8.02 & 19.11 & 0.69 & 0.49 & 23.00 & 25.33 & 0.38 & 0.42 \\
\hline $\mathrm{m} 4$ & 36 & 25.43 & 40.46 & 1.73 & 0.87 & 13.67 & 14.67 & 0.23 & 0.24 \\
\hline $\mathrm{m} 3$ & 31 & 24.60 & 64.47 & 1.04 & 0.40 & 11.33 & 10.67 & 0.19 & 0.18 \\
\hline $\mathrm{m} 2$ & 39 & 19.45 & 34.85 & 0.50 & 0.49 & 13.33 & 17.33 & 0.22 & 0.29 \\
\hline $\mathrm{m} 1$ & 34 & 21.23 & 61.14 & 2.35 & 1.35 & 13.00 & 14.00 & 0.22 & 0.23 \\
\hline f1 & 27 & 4.90 & 9.42 & 0.13 & 0.19 & 19.00 & 21.00 & 0.32 & 0.35 \\
\hline f2 & 27 & 20.02 & 43.24 & 1.95 & 1.38 & 10.33 & 10.50 & 0.17 & 0.18 \\
\hline f3 & 36 & 9.33 & 24.03 & 0.46 & 0.44 & 18.33 & 19.33 & 0.31 & 0.32 \\
\hline $\mathrm{f} 4$ & 33 & 19.44 & 92.40 & 0.24 & 0.84 & 17.33 & 19.67 & 0.29 & 0.33 \\
\hline f5 & 32 & 9.16 & 25.11 & 0.83 & 0.70 & 20.67 & 23.00 & 0.34 & 0.38 \\
\hline Mean & 33.40 & 16.16 & 41.42 & 0.99 & 0.72 & 16.00 & 17.55 & 0.27 & 0.29 \\
\hline st.dev. & 4.30 & 7.51 & 25.07 & 0.76 & 0.40 & 4.25 & 5.03 & 0.07 & 0.08 \\
\hline
\end{tabular}




\section{Appendix E}

\section{Raw Data from Each Participant}

\begin{tabular}{|c|c|c|c|c|c|c|}
\hline Participant & M5 & non-we & ighted $\mathrm{f}$ & & & \\
\hline Trial \# & nw2 & nw7 & nw10 & w1 & w2 & w8 \\
\hline $\begin{array}{l}\text { Start of elbow } \\
\text { extension }\end{array}$ & 24 & 32 & 27 & 25 & 34 & 28 \\
\hline $\begin{array}{l}\text { Start of reversal } \\
\text { flexion }\end{array}$ & 37 & 45 & 42 & 38 & 49 & 42 \\
\hline Start downward & 44 & 54 & 54 & 49 & 60 & 54 \\
\hline $\begin{array}{l}\text { Te max } \\
\text { time to Te max }\end{array}$ & 0.852 & 0.517 & 0.706 & 0.642 & 0.472 & 0.371 \\
\hline (frames) & 34 & 45 & 39 & 38 & 49 & 43 \\
\hline $\begin{array}{l}\text { Ts max } \\
\text { time to Ts max }\end{array}$ & 9.656 & 8.099 & 6.311 & 20.84 & 16.45 & 20.04 \\
\hline (frames) & 35 & 48 & 39 & 40 & 52 & 45 \\
\hline $\begin{array}{l}\text { period (frames) } \\
\text { average period }\end{array}$ & 20 & 22 & 27 & 24 & 26 & 26 \\
\hline (frames) & & 23 & & & 25.33 & \\
\hline average Te max & & 0.692 & & & 0.495 & \\
\hline average Ts max & & 8.022 & & & 19.11 & \\
\hline Participant & M4 & & & & & \\
\hline & & non-we & ighted $\mathrm{f}$ & & & \\
\hline $\begin{array}{l}\text { Trial \# } \\
\text { Start of elhow }\end{array}$ & nw9 & nw5 & nw2 & w9 & w7 & w4 \\
\hline extension & 34 & 24 & 26 & 27 & 26 & 37 \\
\hline $\begin{array}{l}\text { Start of reversal } \\
\text { flexion }\end{array}$ & 42 & 31 & 35 & 33 & 35 & 45 \\
\hline Start downward & 48 & 36 & 41 & 39 & 43 & 52 \\
\hline $\begin{array}{l}\text { Te max } \\
\text { time to Te max }\end{array}$ & 1.923 & 0.074 & 3.202 & 0.031 & 1.241 & 1.335 \\
\hline (frames) & 40 & 29 & 33 & 31 & 34 & 41 \\
\hline $\begin{array}{l}\text { Ts max } \\
\text { time to Ts max }\end{array}$ & 27.24 & 20.73 & 28.32 & 33.2 & 41.03 & 47.16 \\
\hline (frames) & 39 & 28 & 33 & 31 & 34 & 42 \\
\hline period (frames) & 14 & 12 & 15 & 12 & 17 & 15 \\
\hline $\begin{array}{l}\text { average period } \\
\text { (frames) }\end{array}$ & & 13.67 & & & 14.67 & \\
\hline average Te max & & 1.733 & & & 0.869 & \\
\hline average Ts max & & 25.43 & & & 40.46 & \\
\hline Participant & M3 & non-we & ighted $\mathrm{f}$ & & & \\
\hline $\begin{array}{l}\text { Trial \# } \\
\text { Start of elbow }\end{array}$ & nw3 & nw5 & nw9 & w2 & w6 & w8 \\
\hline $\begin{array}{l}\text { extension } \\
\text { Start of reversal }\end{array}$ & 25 & 27 & 17 & 18 & 23 & 24 \\
\hline flexion & 31 & 33 & 22 & 23 & 28 & 29 \\
\hline Start downward & 38 & 38 & 27 & 28 & 34 & 35 \\
\hline
\end{tabular}




\begin{tabular}{|c|c|c|c|c|c|c|}
\hline $\begin{array}{l}\text { Te max } \\
\text { time to Te max } \\
\text { (frames) }\end{array}$ & 1.724 & 0.55 & 0.832 & 0.24 & 0.783 & 0.191 \\
\hline (frames) & 30 & 31 & 21 & 21 & 27 & 28 \\
\hline $\begin{array}{l}\text { Ts max } \\
\text { time to Ts max }\end{array}$ & 24.31 & 20.18 & 29.33 & 66.49 & 67.38 & 59.54 \\
\hline (frames) & 31 & 31 & 21 & 21 & 27 & 28 \\
\hline period (frames) & 13 & 11 & 10 & 10 & 11 & 11 \\
\hline $\begin{array}{l}\text { average period } \\
\text { (frames) }\end{array}$ & & 11.33 & & & 10.67 & \\
\hline average Te max & & 1.035 & & & 0.405 & \\
\hline average Ts max & & 24.6 & & & 64.47 & \\
\hline Participant & M2 & & & & & \\
\hline & & weight & ed first & & & \\
\hline $\begin{array}{l}\text { Trial \# } \\
\text { Start of elbow }\end{array}$ & w12 & w6 & w3 & nw10 & nw4 & nw2 \\
\hline extension & 24 & 36 & 28 & 21 & 20 & 29 \\
\hline $\begin{array}{l}\text { Start of reversal } \\
\text { flexion }\end{array}$ & 34 & 46 & 38 & 27 & 27 & 36 \\
\hline Start downward & 41 & 53 & 46 & 34 & 33 & 43 \\
\hline $\begin{array}{l}\text { Te max } \\
\text { time to Te max }\end{array}$ & 0.793 & 0.466 & 0.204 & 0.349 & 0.381 & 0.764 \\
\hline (frames) & 31 & 43 & 36 & 26 & 25 & 34 \\
\hline $\begin{array}{l}\text { Ts max } \\
\text { time to Ts max }\end{array}$ & 40.76 & 40.71 & 23.08 & 21.12 & 17.4 & 19.84 \\
\hline (frames) & 31 & 44 & 36 & 27 & 25 & 33 \\
\hline $\begin{array}{l}\text { period (frames) } \\
\text { averaqe period }\end{array}$ & 17 & 17 & 18 & 13 & 13 & 14 \\
\hline (frames) & & 17.33 & & & 13.33 & \\
\hline average Te max & & 0.488 & & & 0.498 & \\
\hline average Ts max & & 34.85 & & & 19.45 & \\
\hline Participant & M1 & non-we & ighted 1 & & & \\
\hline Trial \# & nw3 & nw5 & w9 & w5 & & \\
\hline $\begin{array}{l}\text { Start of elbow } \\
\text { extension }\end{array}$ & 22 & 26 & 26 & 20 & & \\
\hline $\begin{array}{l}\text { Start of reversal } \\
\text { flexion }\end{array}$ & 30 & 32 & 33 & 27 & & \\
\hline Start downward & 37 & 37 & 40 & 34 & & \\
\hline $\begin{array}{l}\text { Te max } \\
\text { time to Te max }\end{array}$ & 1.423 & 3.276 & 1.418 & 1.289 & & \\
\hline (frames) & 29 & 31 & 31 & 25 & & \\
\hline $\begin{array}{l}\text { Ts max } \\
\text { time to Ts max }\end{array}$ & 16.01 & 26.46 & 60.32 & 61.96 & & \\
\hline (frames) & 31 & 31 & 31 & 25 & & \\
\hline $\begin{array}{l}\text { period (frames) } \\
\text { average period }\end{array}$ & 15 & 11 & 14 & 14 & & \\
\hline (frames) & 13 & & 14 & & & \\
\hline average Te max & 2.35 & & 1.354 & & & \\
\hline average Ts max & 21.23 & & 61.14 & & & \\
\hline Participant & $\mathrm{F} 1$ & & & & & \\
\hline $\begin{array}{l}\text { Trial \# } \\
\text { Start of elbow }\end{array}$ & ${ }^{w 2}$ & $\begin{array}{l}\text { weight } \\
\text { w7 } \\
\quad 42\end{array}$ & $\begin{array}{l}\text { ed 1st } \\
\text { w10 } \\
43\end{array}$ & $\begin{array}{r}\text { nw1 } \\
39\end{array}$ & $\begin{array}{r}\text { nw5 } \\
45\end{array}$ & $\begin{array}{r}\mathrm{nw} 10 \\
46\end{array}$ \\
\hline
\end{tabular}




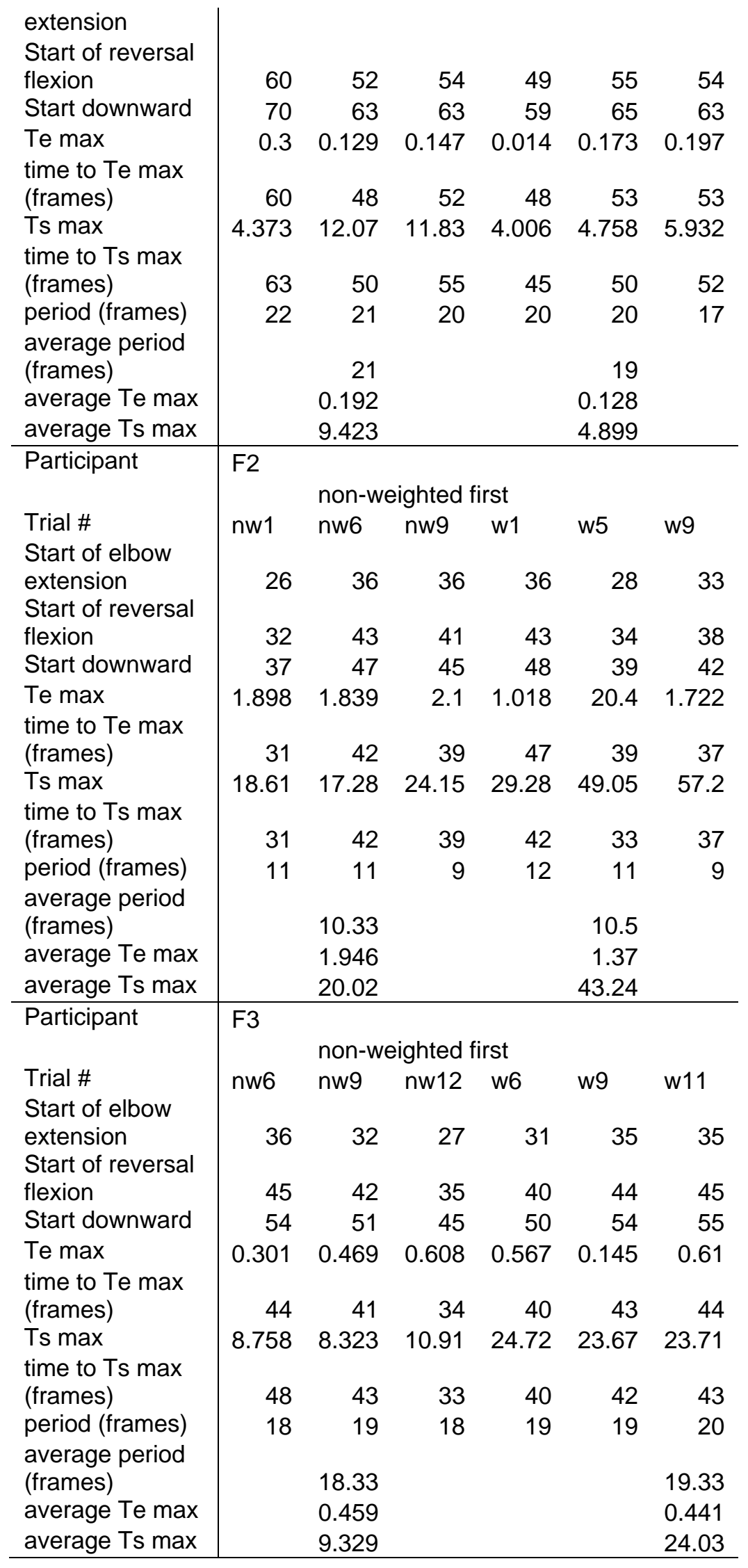




\begin{tabular}{|c|c|c|c|c|c|c|}
\hline Participant & F4 & & non-w & ighted $\mathrm{f}$ & irst & \\
\hline Trial \# & nw4 & nw7 & nw9 & w5 & w7 & w9 \\
\hline $\begin{array}{l}\text { Start of elbow } \\
\text { extension }\end{array}$ & & 40 & 40 & 25 & 31 & 43 \\
\hline $\begin{array}{l}\text { Start of reversal } \\
\text { flexion }\end{array}$ & 52 & 49 & 48 & 37 & 42 & 55 \\
\hline Start downward & 61 & 56 & 55 & 45 & 50 & 63 \\
\hline $\begin{array}{l}\text { Te max } \\
\text { time to Te max }\end{array}$ & 0.18 & 0.475 & 0.077 & 0.743 & 0.922 & 0.863 \\
\hline (frames) & 51 & 47 & 45 & 34 & 40 & 52 \\
\hline $\begin{array}{l}\text { Ts max } \\
\text { time to Ts max }\end{array}$ & 15.99 & 21.7 & 20.61 & 86.2 & 98.34 & 92.65 \\
\hline (frames) & 56 & 46 & 43 & 35 & 41 & 54 \\
\hline $\begin{array}{l}\text { period (frames) } \\
\text { averaqe period }\end{array}$ & 21 & 16 & 15 & 20 & 19 & 20 \\
\hline (frames) & & & 17.33 & & & 19.67 \\
\hline average Te max & & & 0.244 & & & 0.843 \\
\hline average Ts max & & & 19.43 & & & 92.4 \\
\hline Participant & F5 & & & & & \\
\hline & & & non-w & ighted $t$ & irst & \\
\hline $\begin{array}{l}\text { Trial \# } \\
\text { Start olhow }\end{array}$ & nw3 & nw7 & nw10 & w3 & w6 & w9 \\
\hline extension & 40 & 31 & 32 & 36 & 27 & 24 \\
\hline $\begin{array}{l}\text { Start of reversal } \\
\text { flexion }\end{array}$ & 52 & 40 & 42 & 50 & 38 & 35 \\
\hline Start downward & 64 & 49 & 52 & 62 & 50 & 44 \\
\hline $\begin{array}{l}\text { Te max } \\
\text { time to Te max }\end{array}$ & 0.724 & 1.032 & 0.726 & 0.594 & 0.749 & 0.769 \\
\hline (frames) & 45 & 37 & 38 & 44 & 32 & 34 \\
\hline $\begin{array}{l}\text { Ts max } \\
\text { time to Ts max }\end{array}$ & 8.502 & 10.78 & 8.194 & 23.04 & 28.94 & 23.33 \\
\hline (frames) & 43 & 36 & 36 & 57 & 31 & 33 \\
\hline period (frames) & 24 & 18 & 20 & 26 & 23 & 20 \\
\hline $\begin{array}{l}\text { average period } \\
\text { (frames) }\end{array}$ & & & 20.67 & & & 23 \\
\hline average Te max & & & 0.827 & & & 0.704 \\
\hline average Ts max & & & 9.158 & & & 25.11 \\
\hline
\end{tabular}




\section{Appendix F}

Statistical Results of Dependent t-tests:

\begin{tabular}{|l|c|c|c|c|c|c|}
\hline & \multicolumn{2}{|c|}{ Ts (N-m) } & \multicolumn{2}{c|}{ Te (N-m) } & \multicolumn{2}{c|}{$\begin{array}{c}\text { Movement time } \\
\text { (frames) }\end{array}$} \\
\hline & $\begin{array}{c}\text { No } \\
\text { weight }\end{array}$ & Weight & $\begin{array}{c}\text { No } \\
\text { weight }\end{array}$ & Weight & $\begin{array}{c}\text { No } \\
\text { weight }\end{array}$ & Weight \\
\hline Mean & 16.16 & 41.42 & 0.99 & 0.72 & 16.00 & 17.55 \\
\hline Variance & 56.46 & 628.49 & 0.58 & 0.16 & 18.05 & 25.23 \\
\hline Standard Deviation & 7.51 & 25.07 & 0.76 & 0.40 & 4.25 & 5.03 \\
\hline Degrees of Freedom & \multicolumn{2}{|c|}{9} & \multicolumn{2}{c|}{9} & \multicolumn{2}{c|}{0.0049} \\
\hline P two-tail & \multicolumn{2}{|c|}{0.0035} & \multicolumn{2}{c|}{0.1074} & \multicolumn{2}{c|}{2.2622} \\
\hline T critical & \multicolumn{2}{|c|}{2.2622} & \multicolumn{2}{c|}{1.7879} & \multicolumn{2}{c|}{-3.7016} \\
\hline T statistic & \multicolumn{2}{|c|}{-3.920} & \multicolumn{3}{c}{} \\
\hline
\end{tabular}

Note: Statistics generated using Microsoft Excel 2003 Data Analysis package (Microsoft, San Jose, CA). 